\title{
Programa de Expressividade Facial de Emoções e Habilidades Sociais de Crianças Deficientes Visuais e Videntes
}

\author{
Facial Expression of Emotions and Social Skills of Visually Impaired \\ and Sighted Children
}

\author{
Bárbara Carvalho Ferreira* \& Zilda Aparecida Pereira Del Prette \\ Universidade Federal de São Carlos, São Carlos, Brasil
}

\begin{abstract}
Resumo
A expressividade facial de emoções (EFE), tanto em termos topográficos como funcionais, é componente indispensável no desempenho de habilidades sociais (HS). O presente estudo teve como objetivo avaliar um programa de intervenção sobre a EFE e o repertório de habilidades sociais de três crianças cegas, três com baixa visão e três videntes, conforme autoavaliação e avaliação de juiz, pais e professores. Com um delineamento pré e pós-teste com sujeito único, com múltiplas sondagens e replicações intra e entre sujeitos com diferentes graus de comprometimento visual, a intervenção foi realizada ao longo de 21 sessões. Os resultados mostraram mudanças relevantes em termos de melhoria da EFE, bem como seu impacto sobre as classes de habilidades sociais.

Palavras-chave: Expressão facial de emoções, habilidades sociais, intervenção, deficiência visual.
\end{abstract}

\begin{abstract}
The Facial Expression of Emotions (FEE), in its functional and topographic properties, is an essential component of social skills (SS) performance. This study aimed to evaluate a program intervention focused on the FEE of nine children (three blind, three with low vision and three sighted). The intervention impact on children's FEE and their social skills were evaluated by parents, teachers and the children themselves. The intervention was conducted along 21 single-subject sessions, with pre and post evaluation, as well as intra and inter probes and replications among subjects comparing different degrees of visual impairment. Results showed important changes in terms of improving FEE as well as its impact on social skills performance.

Keywords: Facial expression of emotions, social skills, single-subject intervention, visual impairment.
\end{abstract}

No campo das habilidades sociais, a topografia dos comportamentos sociais deve ser analisada a partir de seu impacto sobre a função desses comportamentos, ou seja, na caracterização da qualidade e efetividade das trocas sociais (A. Del Prette \& Del Prette, 2009). Em outras palavras, os componentes da comunicação não-verbal (expressões corporais e faciais, gestualidade, olhar/contato visual, movimentos com a cabeça) são entendidos como indispensáveis para um desempenho socialmente competente (Castanho, Moitrel, Severiano, \& Ribeiro, 2003). Os estudos sobre a relação entre aspectos topográficos e funcionais da comunicação (Philippot, Feldman, \& McGee, 1992; Riggio, 1992) destacam que a proficiência no desempenho dos diferentes componentes não-verbais podem contribuir para o ajustamento social e a satisfação nos relacionamentos (Riggio, 1992; Schachner, Shaver, \& Mikulincer, 2005).

\footnotetext{
* Endereço para correspondência: Centro de Educação e Ciências Humanas, Departamento de Psicologia, Universidade Federal de São Carlos, Relações Interpessoais e Habilidades Sociais, Rodovia Washington Luis, Km 235, Caixa Postal 676, São Carlos, SP, Brasil 13565-905. E-mail: babipocos@yahoo.com.br
}

Dada a relevância dos componentes não-verbais nas relações interpessoais, é necessário que o indivíduo apresente e regule esses componentes em articulação com seus pensamentos, sentimentos e demais ações verbais e motoras, bem como com seus objetivos pessoais e as demandas situacionais e culturais (A. Del Prette \& Del Prette, 2009; Z. A. P. Del Prette \& Del Prette, 2005). Portanto, para se considerar um desempenho como socialmente competente não basta somente a apresentação indiscriminada dos componentes não-verbais, sem o reconhecimento da relevância funcional destes componentes. É necessário, portanto, que o indivíduo reconheça e regule sua expressão facial de forma associada a determinadas classes de habilidades sociais (A. Del Prette \& Del Prette, 2009) e articule seus pensamentos, sentimentos e ações em função de objetivos pessoais e de demandas situacionais e culturais (Z. A. P. Del Prette \& Del Prette, 2005).

A capacidade de expressar emoções de modo a serem reconhecidas por outras pessoas, aqui denominada de expressão diferencial de emoções (EDE), e particularmente, a qualidade da expressividade facial, aqui denominada de expressividade facial de emoções (EFE), envolve o domínio de componentes não-verbais que são críticos para 
a qualidade da interação social (Z. A. P. Del Prette \& Del Prette, 2005). O rosto é o foco principal de ambas (A. Del Prette \& Del Prette, 2001), especialmente diante de demandas que requerem empatia, autocontrole, assertividade e habilidade de fazer amizades. Nesses casos, a expressão facial de emoções depende do correto reconhecimento e nomeação das próprias emoções e da dos outros, dependendo, de algum modo, da capacidade de falar sobre emoções e de lidar com os próprios sentimentos.

A expressão facial das seis emoções básicas (alegria, tristeza, medo, raiva, nojo e surpresa) é inata (Ekman, 1982 , 2004), mas pode ser aprimorada e mantida no decorrer da vida, ou seja, mesmo que inata passa por alterações resultantes da experiência (Otta, Ribeiro, \& Bussab, 2003). Esse aprimoramento pode ocorrer por meio de vários processos mas, particularmente, por observação direta dos desempenhos dos outros e pelo acesso (geralmente visual) aos feedbacks providos pelos demais nas interações. Quando a criança não desenvolve a EFE e a EDE, ou há obstáculos para isso, torna-se necessário programar intervenções visando à manutenção, modulação e aprimoramento das expressões de emoções (Tinti, 2003), em articulação com o repertório de habilidades sociais (Otta, 1994).

Dentre as diferentes populações que podem se beneficiar de programas de promoção EFE e EDE, pode-se destacar as crianças deficientes visuais, cegas e com baixa visão (Anzano \& Rubio, 1995; Tinti, 2003). Segundo dados da literatura (Celeste, 2006; Costa, 2005; D' Allura, 2002), essas crianças apresentam déficits no repertório de habilidades sociais e comprometimentos nas relações interpessoais dadas suas limitações na aprendizagem por observação e dificuldade de acesso aos feedbacks não-verbais dos outros.

No que se refere às expressões faciais destas crianças, estudos apontam que, embora os bebês cegos comecem a sorrir aproximadamente no mesmo período que os bebês videntes e apresentem sorrisos evocados pela voz da mãe, suas expressões faciais vão se tornando mais escassas e menos intensas no decorrer do tempo (Bee, 1996; Ortega, 2003; Otta, 1994). Se, de uma lado, pesquisas indicam que, no geral, as crianças deficientes visuais apresentam alguns comprometimentos na expressão facial de emoções, tanto em termos de repertório menos expressivo como menos regulado e controlado pelas variáveis contextuais e culturais (Castanho, 1996; Castanho et al., 2003; Costa, Del Prette, Cia, \& Del Prette, 2005; Tröster \& Brambring, 1992), do outro lado, há estudos que apontam que o repertório de expressividade facial de emoções das crianças e pessoas deficientes visuais está presente, preservado, e é semelhante ao das pessoas que têm acesso à aprendizagem visual, portanto a modelos e feedbacks não verbais (por exemplo, Galati, Scherer, \& Ricci-Bitti, 1996; Matsumoto \& Willingham, 2009; Tinti, 2003).

As controvérsias dos dados das pesquisas sobre as expressões faciais de emoções das pessoas com deficiência visual podem ser devidas a diversos fatores, como as diferentes metodologias adotadas nos estudos (em termos, por exemplo, de evocação, registro e julgamento deste componente não-verbal), a natureza do estudo e a especificidade do grau de deficiência visual, ou seja, cegueira versus baixa visão. Independentemente dessa controvérsia, alguns pesquisadores (Anzano \& Rubio, 1995; Castanho, 1996; Castanho et al., 2003; Costa, 2005; Godoy, 2007; Otta, 1994; Tinti, 2003) apontam para a importância e necessidade de se planejar intervenções nesta área, associadas ou não ao treinamento de habilidades sociais. Por meio de intervenções específicas, essas crianças poderiam recuperar perdas e aprimorar a EFE e a EDE, em termos de topografia (discriminação dos movimentos faciais associados a cada emoção) e funcionalidade (a relação da expressão facial de emoções com as demandas do contexto e seu efeito sobre as outras pessoas).

Um desafio, no caso das crianças com deficiência visual, é a escassez ou ausência de modelos de intervenção especificamente voltados para a EFE e a EDE, na interface com as habilidades sociais, implicando na necessidade de elaborar e testar procedimentos específicos para isso (Costa, 2005). A elaboração de intervenções efetivas requer, em um primeiro momento, a produção de indicadores de eficácia e efetividade, em termos de validade interna e externa (Z. A. P. Del Prette, \& Del Prette, 2008, 2011). A validade interna depende de delineamentos de pesquisa que permitam estabelecer relação inequívoca entre os resultados do programa e as condições de intervenção, por exemplo, estudos experimentais e replicações sistemáticas (Cozby, 2003; Z. A. P. Del Prette \& Del Prette, 2011). A validade externa ou social depende, amplamente, de indicadores de generalização e manutenção do repertório adquirido, e de seu impacto sobre a vida e o funcionamento psicossocial dos participantes (Z. A. P. Del Prette \& Del Prette, 2008). Além da manutenção e generalização das aquisições, no caso de programas de expressividade emocional, um indicador importante de validade social seria o seu impacto sobre as habilidades sociais e a qualidade das relações interpessoais dos participantes, que deve ser objeto de uma avaliação multimodal com diferentes instrumentos, procedimentos e informantes (Z. A. P. Del Prette \& Del Prette, 2006).

As questões sociais, metodológicas e empíricas antes referidas levaram ao objetivo deste estudo, de avaliar os efeitos de um programa de intervenção sobre o aprimoramento e manutenção da EFE, da EDE e do repertório de habilidades sociais de crianças cegas, com baixa visão e videntes.

\section{Método}

Foi adotado um delineamento pré e pós-teste com sujeito único, com múltiplas sondagens e replicações intra e entre sujeitos com diferentes graus de comprometimento visual, tomando-se como variável independente o programa de intervenção e, como variável dependente, diferentes indicadores de EFE, EDE e habilidades sociais. O delineamento incluiu avaliações pré e pós intervenção conforme ilustrados na Tabela 1. 
Ferreira, B. C. \& Del Prette, Z. A. P. (2013). Programa de Expressividade Facial de Emoções e Habilidades Sociais de Crianças Deficientes Visuais e Videntes.

Tabela 1

Delineamento Pré e Pós-Teste com Sujeito Único, com Múltiplas Sondagens e Replicações Intra e Entre Diferentes Graus de Comprometimento Visual

\begin{tabular}{lcccccccc}
\hline Crianças & Av 1 & Interv & Av 2 & Interv & Av 3 & Interv & Av 4 & Av 5 \\
\hline Cegas & Pré & $\mathrm{X}$ & Pós & & & & & FU \\
Baixa visão & Pré & & Pré & $\mathrm{X}$ & Pós & & & FU \\
Videntes & Pré & & Pré & & Pré & $\mathrm{X}$ & Pós & FU \\
\hline
\end{tabular}

As crianças cegas passaram por um pré-teste, as com baixa visão por dois e os videntes por três. Nas avaliações de pré-teste, como nas de pós e follow-up (seis meses após a intervenção), ocorreram o registro das expressões faciais de emoções e avaliação das HS (autoavaliação e avaliação por pais e professoras). A coleta de dados com os pais e professoras ocorreu somente na avaliação/pré-teste 1 e no pós-teste. Já a coleta com as crianças aconteceu em todas as etapas de avaliação.

Para garantir a validade interna, foram adotados alguns cuidados como: (a) seleção dos participantes com base em repertório deficitário de EFE e habilidades sociais; (b) replicação da intervenção com diferentes participantes, com sondagens pré e pós-teste; (c) avaliação com medidas repetidas ao longo da intervenção; (d) avaliação multimodal com diferentes informantes e métodos; (e) treino de juiz para análise dos dados.

\section{Participantes}

Participaram nove crianças, selecionadas de uma amostra de 29, que foram selecionadas a partir dos seguintes critérios: (a) repertório deficitário de HS no escore global, em pelo menos uma das versões do Sistema de Avaliação das Habilidades Sociais/SSRS-BR (pais, professores e/ou autoavaliação); (b) déficits no uso da face como meio de expressão de emoções, atribuída por pais e professores; (c) qualidade da EFE ruim ou razoável em, no mínimo, três das seis emoções avaliadas por juiz, com base em fotografias.

A amostra final foi composta por três crianças com cegueira congênita (dois meninos e uma menina), três com baixa visão (duas meninas e um menino) e três videntes (dois meninos e uma menina). Os nove participantes deste estudo frequentavam entre a $1^{\mathrm{a}}$ e $4^{\mathrm{a}}$ Série do Ensino Fundamental de escolas públicas no interior dos estados de São Paulo e Minas Gerais. A idade média das crianças era de 8,7 anos $(D P=1,09)$. Participaram, também, como informantes e/ou avaliadores, seus pais, professoras e juiz.

\section{Instrumentos e Materiais}

Sistema de Avaliação de Habilidades Sociais (SSRS-BR). Escala validada para o contexto brasileiro (Bandeira, Del Prette, Del Prette, \& Magalhães, 2009), com propriedades psicométricas satisfatórias. Avalia o repertório de habilidades sociais, comportamentos problemáticos e competência acadêmica de crianças, em versões de autoavaliação (SSRS-criança), de avaliação pelos pais ou responsáveis (SSRS-pais) e pelo professor (SSRS-professor).
Neste estudo foram utilizadas as três versões de avaliação, mas somente para habilidades sociais.

Ficha de Avaliação da EDE por Fotografias e Filmagens Versão Juizes e Ficha de Avaliação da EDE apenas por Fotografias Versão Pais e Professoras. Protocolo, composto por três versões. Na versão juiz, ficha com os códigos das fotografias e filmagens de cada criança (referente à expressão facial de diferentes emoções) dispostos aleatoriamente, com a seguinte instrução: "Olhando para a fotografia/filmagem do rosto dessa criança, o que você acha que ele(a) está sentindo?" Logo abaixo da pergunta, espaço para a resposta do avaliador e para observações que considerar necessárias. Na versão pais e professoras, protocolo de avaliação por escolha com opções (com acesso aos cartões com nomes das emoções), das fotografias da face do(a) seu filho(a) e aluno(a), respectivamente, contendo a seguinte instrução: "Olhando para a fotografia desse rosto, qual destes cartões você acha que corresponde ao que essa criança está sentindo?". Logo abaixo da pergunta, um espaço para a resposta do avaliador e outro para eventuais observações.

Protocolo de Avaliação da Qualidade da Expressividade Facial de Emoção (EFE) por meio de Fotografias. Escala, com alternativas de respostas para cada emoção, que permite avaliar a qualidade da EFE em termos da ausência ou presença de sinais faciais característicos de cada emoção: $0=$ Repertório ruim (não apresenta nenhuma característica clássica na expressão facial de uma determinada emoção); 1 = Repertório razoável (apresenta uma característica clássica na expressão facial de uma determinada emoção); 2 = Repertório bom (apresenta duas características clássicas na expressão facial de uma determinada emoção); 3 = Repertório muito bom (apresenta três características clássicas na expressão facial de uma determinada emoção).

\section{Materiais e Equipamentos Usados na Intervenção}

Máquina fotográfica, filmadora, cartões de identificação das emoções, folhetos instrucionais para pais e professores contendo: (a) descrição das habilidades alvos de cada módulo da intervenção; (b) ênfase na importância de discriminarem a expressão de determinada emoção pela criança (e sua funcionalidade para as relações interpessoais no contexto familiar e escolar); (c) dicas para estimularem o desenvolvimento social e emocional da criança.

Além destes materiais, foram utilizados no presente estudo, materiais produzidos ou organizados especificamente 
para esta pesquisa (Ferreira, 2008, 2012): (a) rostos de bonecos, acolchoados, sendo seis referentes a cada emoção básica, com os principais sinais faciais característicos de cada determinada emoção em alto relevo, com recursos ampliados e contraste de cores. Estes rostos foram confeccionados com diferentes materiais (como, por exemplo, tecido, E.V.A, lã etc.), a partir das descrições dos sinais faciais característicos de cada emoção presentes no livro de Ekman e Friesen (1975); (b) histórias infantis gravadas em áudio, sons e trechos de desenhos infantis; (c) fantoches; (d) materiais diversos utilizados para evocação das expressões faciais de emoções (por exemplo, para evocar alegria, brindes que as crianças indicaram em uma ficha de investigação de possíveis reforçadores, para evocar nojo, objetos com diferentes texturas, odores). Além desses foram também utilizadas vivências adaptadas do livro de Z. A. P. Del Prette e Del Prette (2005). Todos estes materiais foram avaliados em estudo piloto quanto à sua aplicabilidade, por uma professora cega, uma criança cega e outra vidente. As pesquisadoras selecionaram materiais diversos que permitiam às crianças explorarem os diferentes sentidos de olfato, tato, audição, como recomendam Laplane e Batista (2008).

\section{Procedimentos de Coleta de Dados}

Aspectos Éticos. Após aprovação projeto de pesquisa pelo Comitê de Ética da Universidade Federal de São Carlos (UFSCar), foram firmados os acordos da pesquisadora com os pais e professoras e explicitado as questões pertinentes.

Etapas Prévias à Intervenção. Pais, professoras e as crianças responderam a versão correspondente do SSRS-BR, individualmente, em presença da pesquisadora, após as devidas instruções para preenchimento do instrumento.

Nessa etapa foi realizado também o registro por meio de fotografias e filmagem (ambas no mesmo momento) das expressões faciais de emoções das crianças, evocadas diretamente pela pesquisadora, ao longo de uma sequência de procedimentos: (a) apresentação de histórias gravadas em áudio, com sons possíveis de serem associados a determinadas emoções e trechos de filmes ou desenhos cujos personagens expressavam alguma emoção ou cujas variáveis presentes no contexto do filme levassem a associação com um determinado sentimento; (b) conversa com a criança a respeito do sentimento do personagem da história ou das emoções possíveis de serem associadas a determinados sons; (c) retomada de algumas respostas da criança com base no "Roteiro de Avaliação da Expressividade Emocional", relacionadas com a emoção que estava sendo avaliada naquele momento; (d) conversa com a criança sobre situações associadas ao sentimento que estava sendo avaliado; (e) instruções e prompts verbais para a criança apresentar a expressão facial referida pela pesquisadora (exemplo, "A gente mostra no rosto quando estamos alegres. Faça uma cara alegre para eu ver”). As fotografias e filmagens obtidas nesta etapa e as outras tomadas ao longo do programa de intervenção foram utilizadas para avaliação da EFE e EDE.

Programa de Intervenção. O programa de intervenção, conduzido pela própria pesquisadora, teve como objetivo levar as crianças a: discriminar e expressar os sinais característicos de cada emoção na sua própria face; discriminar as condições do ambiente relacionadas com a expressão de cada emoção; estabelecer relação entre a expressão de um sentimento, principalmente pela face, com as classes de habilidades sociais importantes nas relações sociais, sendo algumas delas: empatia, autocontrole, expressividade emocional, assertividade.

Quanto aos aspetos topográficos, estes foram estimulados solicitando-se que a criança identificasse, por meio do tato (todas as crianças), visão (crianças com baixa visão e videntes) e audição, com a descrição verbal das mudanças observadas na face (características de cada emoção), ou seja, explorando todos os sentidos, as expressões no rosto da pesquisadora, que em alguns momentos era neutra e em outros com a expressividade de uma determinada emoção (daquela que estava sendo enfatizada em um módulo ou daquelas que já tinham sido treinadas); solicitação para que a criança discriminasse e descrevesse as mudanças que ocorriam na face da pesquisadora na expressão de cada emoção; solicitação para que a criança identificasse e descrevesse os sinais faciais característicos de cada emoção nos rostos de bonecos confeccionados especialmente para a pesquisa; solicitação para que a criança apresentasse no próprio rosto a expressão de determinada emoção, para, depois disso, iniciar a modelagem da expressão desta emoção na própria face da criança (partindo do repertório de entrada da criança para cada emoção ou das características/sinais mais simples até os mais complexos de serem expressos); solicitação para que a criança apresentasse as expressões faciais de emoções já trabalhadas, para a pesquisadora adivinhar o que ela estava expressando (este procedimento permitiu à pesquisadora rever o que já havia sido trabalhado com a criança e, assim, realizar possíveis revisões); entre outros procedimentos.

No que se referem aos aspectos funcionais, estes foram estimulados por meio de diferentes procedimentos, tais como: (a) exposição oral pela pesquisadora, utilizando teatro com fantoches, de histórias em que os personagens principais expressavam diferentes emoções, com demandas relacionadas às diferentes classes de habilidades sociais e sondagem com as crianças dos estímulos antecedentes e consequentes associados à expressão de cada emoção; (b) criação e representação de histórias relacionadas a cada emoção pela própria criança (todas as histórias eram gravadas em áudio para audição, representação por meio de teatrinhos e discussão posterior com a criança); (c) apresentação de trechos de filmes e desenhos infantis (selecionados pelas próprias crianças) onde o personagem apresentava determinada emoção, seguido de discussão com a criança a respeito das situações representadas na 
Ferreira, B. C. \& Del Prette, Z. A. P. (2013). Programa de Expressividade Facial de Emoções e Habilidades Sociais de Crianças Deficientes Visuais e Videntes.

história (por exemplo, no filme $O$ Rei Leão: “O que Simba está sentido?" "Como identificou que Simba estava triste?" "Por que será que ele está triste, o que aconteceu?" "Quando você fica triste?", entre outras). Muitas das atividades utilizadas focalizaram o "faz-de-conta" que, segundo Hueara, de Souza, Batista, Melgaço e Tavares (2006), permitem elaborações por parte das crianças e desempenho de diferentes capacidades que não seria possível observar em situações mais estruturadas e padronizadas.

O programa foi conduzido em formato individual, com 21 sessões de aproximadamente 60 minutos cada, três e cinco vezes por semana. As sessões apresentavam uma estrutura semelhante, mas com flexibilidade para alterações de procedimentos e materiais adaptados às características, recursos e especificidades da necessidade educacional especial de cada criança. Para as crianças cegas, por exemplo, foram utilizados materiais em alto relevo, com diferentes texturas, com recursos sonoros e impressos em Braille (no caso daquelas já alfabetizadas); para as crianças com baixa visão os materiais eram amplos, com contraste de cores e texturas. Todos estes materiais foram avaliados (estudo piloto), em termos de sua aplicabilidade, por uma professora cega, uma criança cega e outra vidente.

As atividades planejadas para cada sessão seguiram um padrão cumulativo, ou seja, habilidades que foram objeto de sessões anteriores eram recapituladas nos módulos seguintes, visando o aperfeiçoamento e o monitoramento de possíveis déficits adicionais. Os procedimentos, lúdicos e educativos, foram associados a técnicas de manejo de comportamento, dentre elas: reforçamento e reforçamento diferencial, modelagem, imitação e extinção. Para a generalização e manutenção do repertório aprendido, foram adotados os procedimentos de: (a) tarefas de casa; (b) entrega pela pesquisadora dos folhetos instrucionais a pais e professoras, ocasião em que explicava pessoalmente seu conteúdo, esclarecia dúvidas e os estimulava a colaborarem com o programa; (c) telefonemas para os pais, para verificar dúvidas bem como sondar envolvimento e reações aos eventuais impactos do programa. A descrição mais detalhada deste programa de intervenção e dos procedimentos adotados pode ser encontrado em Ferreira (2012).

Avaliações Após a Intervenção - Pós-Teste e FollowUp. Similar às Avaliações Pré-Intervenção.

\section{Tratamento dos Dados}

Os dados dos inventários foram convertidos em escores (para a escala total e para as subescalas) conforme manual de instruções. Para a avaliação da EDE foi entregue aos pais, e professoras, a ficha de avaliação das fotografias nas sondagens de pré-testes, pós-teste e follow-up, e ao juiz a ficha de avaliação da EDE por fotografias e filmagem. Nesta etapa, o juiz também avaliou a EFE das fotografias obtidas nas avaliações pré-teste, pós-teste e follow-up, preenchendo o protocolo elaborado para isso. Os escores no SSRS-BR, juntamente com as pontuações da EFE e da EDE, foram organizados em planilhas no aplicativo SPSS 15.0 for Windows (Statistical Package for Social Sciences) e Excel, sendo analisados por meio de estatística descritiva, gráficos e figuras ilustrativas. Para determinar se as mudanças verificadas na comparação entre pré e pós-teste podem ser atribuídas aos procedimentos utilizados e não por erros de medidas, e significância clínica (permite verificar o grau em que os participantes atingiram os padrões esperados de melhora), foi realizada uma análise com o Método JT (Aguiar, Aguiar, \& Del Prette, 2009) dos dados do SSRS-BR.

\section{Resultados}

Os resultados de cada conjunto de avaliações, em cada momento, são apresentados a seguir. Em cada conjunto, eles foram organizados por subgrupos de crianças, cegas, de baixa visão e videntes.

\section{Expressividade Diferencial de Emoções (EDE)}

A Tabelas 2, 3 e 4 apresentam os resultados da EDE dos participantes cegos, de baixa visão e videntes, respectivamente, nas sondagens de pré-testes, pós-teste e follow-up, conforme avaliação por juiz, pais e professoras.

Tabela 2

Expressões Faciais de Emoções das Crianças Cegas (P1, P2 e P3) Avaliadas por Juiz, Pais e Professoras

\begin{tabular}{|c|c|c|c|c|c|c|c|c|c|}
\hline \multirow{2}{*}{ Emoção } & \multicolumn{3}{|c|}{$\mathrm{P} 1 \overbrace{}^{\lambda}$} & \multicolumn{3}{|c|}{$\mathrm{P} 2 \overbrace{}^{\lambda}$} & \multicolumn{3}{|c|}{$\mathrm{P} 3$ ㅇ } \\
\hline & Av. 1 & Pós & FU & Av. 1 & Pós & FU & Av. 1 & Pós & FU \\
\hline Alegria & $\boldsymbol{v} \square \bullet \boldsymbol{\Delta}$ & $\boldsymbol{\square} \square \bullet \boldsymbol{\Delta}$ & $\boldsymbol{\square} \square \bullet \boldsymbol{\Delta}$ & $\square$ & $\boldsymbol{\square} \square \bullet \boldsymbol{\Delta}$ & $\boldsymbol{\square} \square \bullet \boldsymbol{\Delta}$ & $\mathbf{\square} \bullet \boldsymbol{\Delta}$ & $\mathbf{m} \square \bullet \boldsymbol{\Delta}$ & $\boldsymbol{\square} \square \bullet \boldsymbol{\Delta}$ \\
\hline Tristeza & $\| \Delta$ & $\boldsymbol{\|} \square \bullet \boldsymbol{\Delta}$ & $\mathbf{m} \square \boldsymbol{\Delta}$ & $\square \boldsymbol{\Delta}$ & $\mathbf{\square} \square \boldsymbol{\Delta}$ & $\boldsymbol{m} \bullet \boldsymbol{\Delta}$ & $\mathbf{\square} \bullet$ & $\mathbf{\square} \square \boldsymbol{\Delta}$ & $\boldsymbol{\square} \square \boldsymbol{\Delta}$ \\
\hline Medo & & $\boldsymbol{\|} \bullet \boldsymbol{\Delta}$ & $\mathbf{\square} \square \boldsymbol{\Delta}$ & & $\boldsymbol{\square} \square \bullet \boldsymbol{\Delta}$ & $\boldsymbol{m} \square \boldsymbol{\Delta}$ & $\bullet$ & $\square \bullet \boldsymbol{\Delta}$ & $\boldsymbol{\square} \square \bullet \boldsymbol{\Delta}$ \\
\hline Raiva & & $\boldsymbol{\|} \square \boldsymbol{\Delta}$ & $\mathbf{\square} \bullet \boldsymbol{\Delta}$ & & $\boldsymbol{\square} \square \boldsymbol{\Delta}$ & $\| \boldsymbol{\Delta}$ & & $\mathbf{\|} \square \bullet$ & $\boldsymbol{\|} \square \boldsymbol{\Delta}$ \\
\hline Nojo & & $\boldsymbol{\square} \square \boldsymbol{\Delta}$ & $\mathbf{m} \square \boldsymbol{\Delta}$ & $\mathbf{m}$ & $\boldsymbol{\square} \square \bullet \boldsymbol{\Delta}$ & $\boldsymbol{m} \square \boldsymbol{\Delta}$ & & $\mathbf{m} \square \bullet \boldsymbol{\Delta}$ & $\mathbf{m} \square \boldsymbol{\Delta}$ \\
\hline Surpresa & & $\square \bullet \boldsymbol{\Delta}$ & $\square \bullet \boldsymbol{\Delta}$ & & $\boldsymbol{\|} \square \boldsymbol{\Delta}$ & $\boldsymbol{\|} \bullet \boldsymbol{\Delta}$ & & $\boldsymbol{\|} \bullet \bullet$ & $\square \bullet \boldsymbol{\Delta}$ \\
\hline
\end{tabular}

Nota . + = Sexo feminino; $\widehat{\delta}=$ Sexo masculino; Avaliação juiz fotografia $=\mathbf{m}$; Avaliação juiz filmagem $=\square ;$ Pais $=\bullet$ • Profa. $=\boldsymbol{\Lambda}$. 
Observa-se que os avaliadores foram bastante consistentes em suas avaliações da EDE das crianças cegas, tanto antes como depois da intervenção. As expressões faciais inicialmente identificadas foram basicamente de alegria e tristeza, com o juiz acrescentando nojo para P2 e uma mãe identificando a expressão de medo de sua filha
(P3). Na avaliação pós-intervenção, todas as seis emoções básicas foram identificadas, pelos três julgadores, ou por pelo menos por dois deles. Na avaliação de follow-up, seis meses após a intervenção, praticamente todas as aquisições se mantiveram. Além disso, observa-se pouca diferença na avaliação do juiz das EDE registradas por fotografia e filmagem.

Tabela 3

Expressões Faciais de Emoções das Crianças com Baixa Visão (P4, P5 e P6) Avaliadas por Juiz, Pais e Professoras

\begin{tabular}{|c|c|c|c|c|c|c|c|c|c|c|c|c|}
\hline \multirow[t]{2}{*}{ Emoção } & \multicolumn{4}{|c|}{$\mathrm{P} 4 \hat{\sigma}$} & \multicolumn{4}{|c|}{ P5 } & \multicolumn{4}{|c|}{ P6 9} \\
\hline & Av.1 & Av.2 & Pós & FU & Av. 1 & Av. 2 & Pós & FU & Av.1 & Av. 2 & Pós & FU \\
\hline Alegria & $\mathbf{\square} \bullet \boldsymbol{\Delta}$ & $\boldsymbol{\square} \square \boldsymbol{\Delta}$ & $\mathbf{\square} \bullet \boldsymbol{\Delta}$ & $\mathbf{\square} \bullet \boldsymbol{\Delta}$ & $\boldsymbol{\square} \square \boldsymbol{\Delta}$ & $\mathbf{m} \square \boldsymbol{\Delta}$ & $\mathbf{m} \Delta$ & - & $\mathbf{\square} \bullet \boldsymbol{\Delta}$ & $\mathbf{\square} \bullet \boldsymbol{\Delta}$ & $\mathbf{\square} \bullet \boldsymbol{\Delta}$ & $\boldsymbol{\square} \square \boldsymbol{\Delta}$ \\
\hline Tristeza & $\mathbf{m}$ & & $\boldsymbol{\nabla} \square \bullet \boldsymbol{\Delta}$ & $\boldsymbol{\nabla} \square \bullet \boldsymbol{\Delta}$ & & $\mathbf{\square}$ & $\boldsymbol{\square} \square \boldsymbol{\Delta}$ & - & $\bullet \boldsymbol{\Delta}$ & & $\boldsymbol{\square} \bullet \boldsymbol{\Delta}$ & $\mathbf{v} \bullet \boldsymbol{\Delta}$ \\
\hline Medo & & & $\square \bullet \boldsymbol{\Delta}$ & $\mathbf{m}$ & & & $\mathbf{m}$ & - & & & $\boldsymbol{\Delta} \square \boldsymbol{\Delta} \Delta$ & $\mathbf{v} \bullet \mathbf{\Delta}$ \\
\hline Raiva & $\bullet$ & $\boldsymbol{\square} \square \boldsymbol{\Delta}$ & $\boldsymbol{\nabla} \square \bullet \mathbf{\Delta}$ & $\boldsymbol{\nabla} \square \bullet \mathbf{\Delta}$ & & & $\boldsymbol{\Delta} \Delta$ & - & घ & & $\boldsymbol{\Delta} \square \boldsymbol{\Delta} \Delta$ & $\mathbf{v} \bullet \mathbf{\Delta}$ \\
\hline Nojo & & $\boldsymbol{\square} \square \boldsymbol{\Delta}$ & $\boldsymbol{\nabla} \square \bullet \mathbf{\Delta}$ & $\mathbf{D} \square \boldsymbol{\Delta}$ & $\mathbf{v} \boldsymbol{\Delta}$ & $\mathbf{\square} \square \boldsymbol{\Delta}$ & $\boldsymbol{\nabla} \square \boldsymbol{\Delta}$ & - & $\mathbf{D} \square \boldsymbol{\Delta}$ & $\boldsymbol{n} \square \bullet \boldsymbol{\Delta}$ & $\boldsymbol{\square} \bullet \boldsymbol{\Delta}$ & $\boldsymbol{v} \square \bullet \boldsymbol{\Delta}$ \\
\hline Surpresa & & & $\mathbf{D} \square \boldsymbol{\Delta}$ & $\square \bullet \boldsymbol{\Delta}$ & & & $\mathbf{\square} \boldsymbol{\Delta}$ & - & & & $\boldsymbol{\square} \square \boldsymbol{\Delta}$ & $\mathbf{\square} \bullet \boldsymbol{\Delta}$ \\
\hline
\end{tabular}

Nota $. q=$ Sexo feminino; $\widehat{\sigma}=$ Sexo masculino; Não realizou = -; Avaliação juiz fotografia = $\mathbf{m}$; Avaliação juiz filmagem = $\square$; Pais $=\bullet$; Profa. $=\boldsymbol{\Delta}$.

Pode-se verificar que nas avaliações que antecederam a intervenção, mais expressões faciais de emoções foram identificadas na face das crianças com baixa visão, comparativamente com as crianças cegas. Além de alegria e tristeza, raiva (para P4 e P6) e nojo (P4, P5 e P6) foram identificadas por pelo menos um dos avaliadores. Na sondagem logo após a intervenção e no follow-up, a expressão facial das seis emoções básicas foi identificada nos três participantes com baixa visão, pela maioria dos avaliadores. Em 93,9\% dos dados houve concordância entre os julgamentos do juiz por fotografia e filmagem.

Tabela 4

Expressões Faciais de Emoções das Crianças Videntes (P7, P8 e P9) Avaliadas por Juiz, Pais e Professoras

\begin{tabular}{|c|c|c|c|c|c|c|c|c|c|c|c|c|c|c|c|}
\hline \multirow{2}{*}{ Emoção } & \multicolumn{5}{|c|}{ P7 0} & \multicolumn{5}{|c|}{$\mathrm{P} 8 \sigma^{\lambda}$} & \multicolumn{5}{|c|}{$\mathrm{P} 9$ 우 } \\
\hline & Av.1 & Av. 2 & Av. 3 & Pós & FU & Av.1 & Av. 2 & Av. 3 & Pós & FU & Av. 1 & Av. 2 & Av. 3 & Pós & FU \\
\hline Alegria & $\bullet \bullet \boldsymbol{\Delta}$ & $\bullet \bullet \bullet \Delta$ & $\bullet \bullet \bullet \Delta$ & $\bullet \bullet \bullet$ & $\bullet \bullet \bullet \Delta$ & $\bullet \bullet \bullet$ & $\bullet \bullet \bullet \boldsymbol{\Delta}$ & $\bullet \bullet \bullet \boldsymbol{\Delta}$ & $\bullet \bullet \bullet$ & $\boldsymbol{-} \square \bullet \boldsymbol{\Delta}$ & $\boldsymbol{m} \bullet \boldsymbol{\Delta}$ & $\bullet \bullet \bullet \Delta$ & $\bullet \bullet \bullet \Delta$ & $\boldsymbol{\square} \bullet \boldsymbol{\Delta}$ & $\boldsymbol{- \square} \bullet \boldsymbol{\Delta}$ \\
\hline Tristeza & & & $\bullet \boldsymbol{\Delta}$ & $\boldsymbol{\square} \square \boldsymbol{\Delta}$ & $\boldsymbol{\square} \bullet \boldsymbol{\Delta}$ & & & $\square \bullet$ & $\boldsymbol{\square} \bullet \boldsymbol{\Delta}$ & $\boldsymbol{\|} \square \boldsymbol{\Delta}$ &.$\square \bullet \boldsymbol{\Delta}$ & & & $\square \bullet \boldsymbol{\Delta}$ & $\boldsymbol{n} \square \boldsymbol{\Delta} \Delta$ \\
\hline Medo & & & & $\mathbf{\square} \bullet \boldsymbol{\Delta}$ & $\boldsymbol{\square} \square \boldsymbol{\Delta}$ & & & & $\boldsymbol{\square} \bullet \boldsymbol{\Delta}$ & $\mathbf{\square} \bullet \boldsymbol{\Delta}$ & & & & $\square \Delta$ & 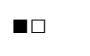 \\
\hline Raiva & $\boldsymbol{\square} \square$ & $\boldsymbol{\square} \bullet \boldsymbol{\Delta}$ & $\boldsymbol{\square} \square \boldsymbol{\Delta}$ & $\boldsymbol{\square} \bullet \boldsymbol{\Delta}$ & $\boldsymbol{\square} \square \boldsymbol{\Delta}$ & & $\square \bullet \boldsymbol{\Delta}$ & $\boldsymbol{\square} \bullet \boldsymbol{\Delta}$ & $\boldsymbol{m} \square \boldsymbol{\Delta}$ & $\boldsymbol{m} \square \boldsymbol{\Delta} \Delta$ & & & & $\square \bullet \boldsymbol{\Delta}$ & $\mathbf{m} \bullet \Delta$ \\
\hline Nojo & $\square \bullet \boldsymbol{\Delta}$ & $\boldsymbol{\square} \bullet \boldsymbol{\Delta}$ & $\boldsymbol{m} \bullet \boldsymbol{\Delta}$ & $\boldsymbol{m} \bullet \boldsymbol{\Delta}$ & $\boldsymbol{m} \bullet \boldsymbol{\Delta}$ & & & $\square \bullet \boldsymbol{\Delta}$ & $\boldsymbol{\square} \bullet \boldsymbol{\Delta}$ & $\boldsymbol{\|} \square \boldsymbol{\Delta}$ & $m$ & $\square \Delta$ & $\boldsymbol{m} \square \boldsymbol{\Delta}$ & $\boldsymbol{m} \square \boldsymbol{\Delta}$ & $\boldsymbol{m} \square \boldsymbol{\Delta} \Delta$ \\
\hline Surpresa & $\square$ & $\square$ & $\square$ & $\boldsymbol{m} \bullet \boldsymbol{\Delta}$ & $\boldsymbol{m} \bullet \boldsymbol{\Delta}$ & & & & $\boldsymbol{m} \square \boldsymbol{\Delta}$ & $\boldsymbol{\|} \square \boldsymbol{\Delta}$ & & & & $\boldsymbol{\square} \square \boldsymbol{\Delta}$ & $\mathbf{m}_{\square} \bullet$ \\
\hline
\end{tabular}

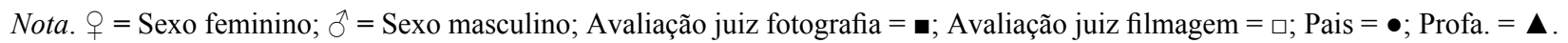

Os dados das crianças videntes assemelham-se aos das crianças com baixa visão: alegria, tristeza, raiva e nojo foram emoções identificadas por um ou mais avaliadores em pelo menos uma das avaliações prévias à intervenção. Nos dados de pré-testes observa-se também uma maior sensibilidade do registro da expressão facial de surpresa de
P7 por filmagem, quando comparado com o julgamento do juiz por fotografia. Nas avaliações pós-intervenção todas as emoções foram identificadas, pelos três avaliadores (P7 e P8) ou por dois deles (P9). No follow-up essa tendência se manteve para pelo menos dois avaliadores, exceto o medo, identificado apenas pelo juiz na criança P9. 
Ferreira, B. C. \& Del Prette, Z. A. P. (2013). Programa de Expressividade Facial de Emoções e Habilidades Sociais de Crianças Deficientes Visuais e Videntes.

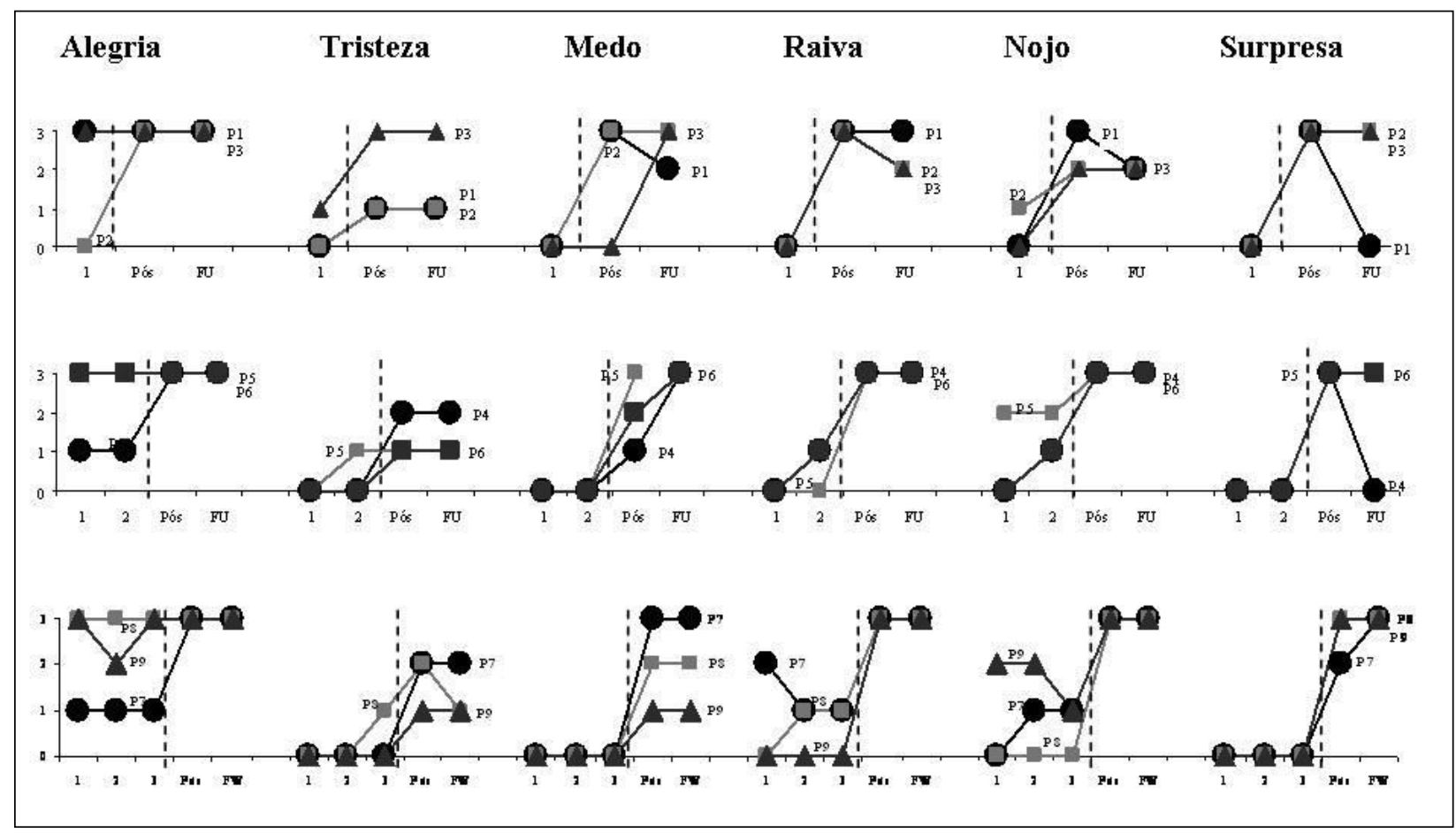

Figura 1. Qualidade das EFE dos nove participantes, nas sondagens de Pré-teste 1, 2 e 3, Pós-teste e Follow-up, conforme avaliação por juiz com base em fotografias.

Qualidade da Expressividade Facial de Emoções (EFE)

Os resultados da EFE, analisadas por um juiz, com base nas fotografias, são apresentados na Figura 1, cuja escala varia de 0 (ruim) a 3 (muito bom).

De modo geral, observa-se uma melhora na qualidade da EFE em seguida à intervenção, em todos os subgrupos de crianças e para praticamente todas as emoções avaliadas. A avaliação de follow-up mantém ou melhora a EFE, com poucas exceções. Em uma análise mais específica, por emoção, verificou-se que:

- Alegria. A EFE inicial já era razoável ou muito boa para oito dos nove participantes. No pós-teste e follow-up todos os participantes atingiram o ápice da qualidade na expressividade de alegria pela face.

- Tristeza. Oito participantes apresentavam inicialmente EFE ruim e um deles apenas razoável. Nas avaliações de pós-intervenção e follow-up observou-se uma melhora da EFE de todos os participantes, especialmente de P3 (cego) e os resultados foram mantidos no follow-up para todos os participantes, exceto P8 (vidente) que passou de EFE boa para razoável, ainda assim, acima da EFE inicial.

- Medo. Todos os participantes apresentavam inicialmente EFE ruim e melhoraram após a intervenção, com destaque para P2, P3 (cegos), P5 (baixa visão) e P7 (vidente). Na avaliação de follow-up, essa melhora foi mantida para cinco participantes e aprimorada para três deles.
- Raiva. Com exceção de P7, todos participantes apresentavam inicialmente EFE ruim ou apenas razoável, mas melhoraram após a intervenção, principalmente as crianças cegas, P5 (baixa visão) e P9 (vidente). As melhoras foram mantidas no follow-up para sete dos nove participantes, com pequena redução de P2 e P3 (cegos), mas ainda acima da EFE inicial.

- Nojo. Seis dos nove participantes apresentavam, nas avaliações pré-intervenção, uma EFE razoável ou boa. Apenas P1 e P3 (cegos) e P8 (vidente) apresentavam EFE ruim, mas todos melhoraram e mantiveram as aquisições no follow-up, destacando-se P4, P6 (baixa visão) e os três videntes.

- Surpresa. Os nove participantes foram avaliados inicialmente com EFE ruim; na pós-intervenção oito passaram para EFE muito boa e um (P7, vidente) para boa. Com exceção de P1 (cego) e P4 (baixa visão), todos mantiveram a EFE elevada, aprimoramento para P7 (vidente).

\section{Habilidades Sociais}

Os resultados referentes ao repertório de habilidades sociais das crianças, avaliado pelos próprios participantes, seus pais e professoras nas sondagens de pré-teste, imediatamente anterior à intervenção, e pós-teste, são apresentados a seguir. Na Tabela 5 são ilustradas as pontuações gerais e por fatores, conforme os três avaliadores. 
Psicologia: Reflexão e Crítica, 26(2), 327-338.

\section{Tabela 5}

Diferença entre Pré-Teste e Pós-Teste na Pontuação Geral e por Fatores de Habilidades Sociais das Crianças Cegas (P1, P2, P3), com Baixa Visão (P4, P5, P6) e Videntes (P7, P8 e P9) no SSRS-BR

\begin{tabular}{|c|c|c|c|c|c|c|c|c|c|c|}
\hline & \multirow{3}{*}{ Versão/fator } & \multicolumn{9}{|c|}{ Avaliadores } \\
\hline & & \multicolumn{3}{|c|}{ Cegos } & \multicolumn{3}{|c|}{ Baixa visão } & \multicolumn{3}{|c|}{ Videntes } \\
\hline & & $\mathrm{P} 1 \hat{0}$ & $\mathrm{P} 2 \hat{\jmath}$ & $\mathrm{P} 3$ q & $\mathrm{P} 4 \hat{\jmath}$ & $\mathrm{P} 50$ & $\mathrm{P} 6 \hat{0}$ & $\mathrm{P} 7 \hat{\jmath}$ & $\mathrm{P} 8{ }^{\lambda}$ & $\mathrm{P} 9$ ㅇ \\
\hline \multirow[t]{7}{*}{ Crianças } & Pontuação geral & +60 & +40 & +50 & +15 & +25 & +70 & +50 & +45 & +90 \\
\hline & Responsabilidade & +50 & +45 & +25 & +20 & $=$ & +30 & +20 & +10 & +10 \\
\hline & Empatia & +20 & +10 & +10 & +5 & +85 & +85 & +35 & +10 & +65 \\
\hline & Assertividade & +25 & +20 & +20 & +10 & +10 & +25 & $=$ & +20 & +65 \\
\hline & Autocontrole & +20 & +20 & +10 & +10 & -10 & +70 & +90 & +60 & +60 \\
\hline & Evit. problemas & +60 & -15 & +30 & +20 & +45 & +80 & +80 & +20 & +50 \\
\hline & Exp. sent. positivo & -5 & -5 & -5 & +5 & +20 & +60 & +10 & +10 & +60 \\
\hline \multirow[t]{7}{*}{ Pais } & Pontuação geral & +25 & +55 & +20 & +10 & +40 & +20 & -20 & +25 & +65 \\
\hline & Cooperação & +5 & $=$ & $=$ & -10 & +5 & +10 & -10 & +20 & +25 \\
\hline & Amabilidade & +20 & +10 & +10 & +30 & -10 & +30 & -10 & +25 & +20 \\
\hline & Iniciativa & +10 & +10 & +20 & $=$ & $=$ & +20 & -10 & +1 & +15 \\
\hline & Asserção & +25 & +55 & +35 & $=$ & +35 & -10 & +4 & +20 & +40 \\
\hline & Autocontrole/civ. & +30 & +25 & +45 & $=$ & +55 & +90 & -30 & +25 & +55 \\
\hline & Autocontrole pass. & +98 & +50 & +30 & +30 & +50 & +20 & +20 & +15 & +30 \\
\hline \multirow[t]{6}{*}{ Professor } & Pontuação geral & +20 & +30 & +20 & +35 & +15 & $=$ & +5 & +25 & +60 \\
\hline & Responsabilidade & +15 & +30 & +20 & +20 & +25 & $=$ & +10 & +20 & +30 \\
\hline & Asserção positiva & -6 & +20 & +10 & +40 & +15 & -5 & +8 & +15 & +30 \\
\hline & Autocontrole & +82 & +30 & +40 & +45 & +35 & +25 & +15 & +20 & +75 \\
\hline & Autodefesa & -10 & +10 & -10 & +5 & +5 & +5 & +10 & +10 & +70 \\
\hline & Cooperação pares & $=$ & +50 & +5 & +10 & +10 & -8 & +5 & +50 & +50 \\
\hline
\end{tabular}

Os dados da Tabela 5 mostram que, analisando as diferenças entre pré e pós-teste, ocorreram ganhos em 151 dos 180 escores produzidos $(83,8 \%)$. Todos os participantes, exceto P7 (avaliação pais), obtiveram ganhos na pontuação geral, evidenciando o aprimoramento após a intervenção. $\mathrm{Na}$ autoavaliação de todas as crianças, o escore global de habilidades sociais melhorou após a intervenção.

Uma análise do repertório de habilidades sociais por fatores ou subescalas indica que, na autoavaliação, o repertório de habilidades sociais das crianças, nos seis fatores do SSRS-BR, foi, em geral, aprimorado após a intervenção. Para algumas crianças, os ganhos em determinadas classes de habilidades sociais foram mais significativos, em termos de mudanças entre um posto percentil mais baixo para mais alto, podendo se destacar: (a) responsabilidade para duas crianças cegas (P1 e P2) e uma com baixa visão (P6); (b) empatia para duas crianças com baixa visão (P5 e P6), duas videntes (P7 e P9), uma cega (P1); (c) evitação de problemas para P1, (cego), P6 (baixa visão) e P7, (vidente); (d) expressão de sentimento positivo para um participante com baixa visão (P6) e dois videntes (P8 e P9).

$\mathrm{Na}$ perspectiva dos pais, os dados indicam, que para exceto P7 (vidente), aprimoramentos nos diferentes fatores avaliados. Por fim, a avaliação das professoras aponta melhoras e manutenção das aquisições nas habilidades sociais de todas as crianças, com exceção de algumas subescalas isoladas que apresentaram redução nos escores: Asserção positiva par P1 (cego) e P6 (baixa visão); 
Ferreira, B. C. \& Del Prette, Z. A. P. (2013). Programa de Expressividade Facial de Emoções e Habilidades Sociais de Crianças Deficientes Visuais e Videntes.

Autodefesa para P1 e P3 (cegos); Cooperação com pares para P6 (baixa visão).
Os dados do SSRS-BR também foram analisados pelo Método JT (Aguiar et al., 2009) e dispostos na Tabela 6.

Tabela 6

Mudança Positiva Confiável e Mudança Clínica na Pontuação Geral e por Fatores de Habilidades Sociais das Crianças Cegas (P1, P2, P3), com Baixa Visão (P4, P5, P6) e Videntes (P7, P8 e P9) no SSRS-BR

\begin{tabular}{|c|c|c|c|c|c|c|c|c|c|c|}
\hline & \multirow{3}{*}{ Versão/Fator } & \multicolumn{9}{|c|}{ Avaliadores } \\
\hline & & \multicolumn{3}{|c|}{ Cegos } & \multicolumn{3}{|c|}{ Baixa visão } & \multicolumn{3}{|c|}{ Videntes } \\
\hline & & $\mathrm{P} 1 \circlearrowleft$ & $\mathrm{P} 2 \widehat{\sigma}$ & $\mathrm{P} 3 \stackrel{+}{\circ}$ & $\mathrm{P} 4 \widehat{\jmath}$ & P5 ત̂ & P6 ठ & P7 ठ઼ & P8 તَ & P9 \\
\hline \multirow[t]{7}{*}{ Crianças } & Pontuação geral & $\mathrm{MPC}^{*}$ & MPC & MPC* & - & - & MPC* & MPC & MPC* & MPC* \\
\hline & Responsabilidade & $\mathrm{MPC}^{*}$ & $\mathrm{MPC}^{*}$ & - & - & - & $\mathrm{MPC}^{*}$ & - & - & - \\
\hline & Empatia & MPC & - & - & - & MPC* & $\mathrm{MPC}^{*}$ & MPC & - & MPC \\
\hline & Assertividade & - & - & - & - & - & - & - & - & $\mathrm{MPC}^{*}$ \\
\hline & Autocontrole & - & - & - & - & - & $\mathrm{MPC}^{*}$ & $\mathrm{MPC}^{*}$ & - & - \\
\hline & Evit. problemas & $\mathrm{MPC}^{*}$ & - & - & - & - & $\mathrm{MPC}^{*}$ & $\mathrm{MPC}^{*}$ & - & - \\
\hline & Exp. sent. posit. & - & - & - & - & - & $\mathrm{MPC}^{*}$ & - & $\mathrm{MPC}^{*}$ & $\mathrm{MPC}^{*}$ \\
\hline \multirow[t]{7}{*}{ Pais } & Pontuação geral & $\mathrm{MPC}^{*}$ & $\mathrm{MPC}^{*}$ & - & - & - & MPC & - & $\mathrm{MPC}^{*}$ & $\mathrm{MPC}$ \\
\hline & Cooperação & $\mathrm{MPC}^{*}$ & - & - & - & - & - & - & - & - \\
\hline & Amabilidade & - & - & - & - & - & - & - & $\mathrm{MPC}^{*}$ & - \\
\hline & Iniciativa & - & - & - & - & - & - & - & - & $\mathrm{MPC}^{*}$ \\
\hline & Asserção & - & MPC & - & - & - & - & - & $\mathrm{MPC}^{*}$ & $\mathrm{MPC}^{*}$ \\
\hline & Autocontrole/civ & - & - & - & - & - & MPC* & - & - & - \\
\hline & Autocontrole pas. & $\mathrm{MPC}^{*}$ & MPC & & - & - & - & - & - & $\mathrm{MPC}$ \\
\hline \multirow[t]{6}{*}{ Professor } & Pontuação geral & MPC & MPC & MPC & $\mathrm{MPC}^{*}$ & $\mathrm{MPC}^{*}$ & - & MPC & $\mathrm{MPC}^{*}$ & $\mathrm{MPC}^{*}$ \\
\hline & Responsabilidade & $\mathrm{MPC}^{*}$ & MPC & MPC* & MPC & $\mathrm{MPC}^{*}$ & - & - & MPC & MPC \\
\hline & Asserção pos. & - & - & - & MPC* & & - & - & MPC* & $\mathrm{MPC}^{*}$ \\
\hline & Autocontrole & $\mathrm{MPC}^{*}$ & MPC & $\mathrm{MPC}^{*}$ & MPC* & MPC* & MPC & MPC & MPC & $\mathrm{MPC}^{*}$ \\
\hline & Autodefesa & - & - & - & - & - & - & - & - & $\mathrm{MPC}^{*}$ \\
\hline & Coop. pares & - & MPC* & - & - & - & - & - & MPC & MPC \\
\hline
\end{tabular}

Nota MPC = Mudança positiva confiável (melhora); $*$ mudança clínica e significativa; MNC = Mudança negativa confiável (piora); - = Ausência de mudança confiável.

$\mathrm{Na}$ análise destes dados, escore geral, pelo Método JT (Aguiar et al., 2009) observou-se que todos os nove participantes apresentaram em uma ou mais versão do SSRS-BR mudança positiva confiável, ou seja, confiabilidade em que as mudanças verificadas na comparação entre pré e pós-teste podem ser atribuídas a intervenção e não por erros de medidas e, que destes nove, oito (exceto P7) apresentaram mudança clínica e significante na perspec- tiva de um ou mais avaliador, passando de uma amostra disfuncional para uma funcional.

Ainda quanto à análise pelo Método JT (Aguiar et al., 2009), mas para cada fator, observou-se que em todos os fatores, das três versões do SSRS-BR, ocorreu mudança positiva confiável (melhora) e mudança clínica e significativa para uma ou mais crianças, com destaque para os fatores responsabilidade, empatia, evitação de problemas e 
expressão de sentimentos positivos na versão auto-avaliação, em que três participantes ou mais apresentaram MPC* e MPC, e para as crianças P1, P6, P7 e P9 que apresentaram MPC* e/ou MPC em três fatores ou mais. No SSRS-BR versão pais e professores também observou-se MPC* e/ ou MPC em todos os fatores para pelo menos uma criança, destacando-se os fatores asserção e autocontrole passivo, e o desempenho das crianças P1, P2, P8 e P9 na versão dos pais; na versão professores, os fatores responsabilidade $\mathrm{e}$ autocontrole, sendo que neste último ocorreu MPC* e/ou MPC para todos os participantes, e o desempenho de P8 e P9. Observa-se assim, que tanto para pontuação geral como para cada fator, nas três versões do instrumento, ocorreram muitas mudanças positivas confiáveis (relacionada com a validade interna do estudo) e mudanças clínicas significativas (relacionada com a significância clínica, em termos da validade externa do estudo).

Analisando os dados da Tabela 6, não observa-se nenhuma mudança negativa confiável/piora (Aguiar et al., 2009), mas ausência de mudança confiável devido ao efeito de teto, ou seja, no pré-teste o repertório de habilidades sociais era alto em determinados fatores para algumas crianças. Assim, de um total de 180 escores produzidos, $38,8 \%$ dos dados foram de mudança positiva confiável e/ou mudança clínica e significativa, e $45 \%$ foram de oscilações positivas dos resultados, ainda que não necessariamente caracterizadas como confiáveis, ou seja, como mudanças.

\section{Discussão}

A intervenção realizada foi planejada para aprimorar as expressões faciais de emoções das crianças, considerando-se a sua importância para a competência em habilidades sociais e no relacionamento interpessoal em geral. $\mathrm{O}$ foco principal foi, portanto, o aperfeiçoamento dos aspectos topográficos e funcionais deste componente não-verbal. A intervenção envolveu o estabelecimento de condições estruturadas para promover a discriminação e apresentação dos movimentos faciais associados a cada emoção e para associar a expressão diferencial de emoções (EDE) e a qualidade da expressividade facial (EFE) com as demandas do contexto, visando seu efeito sobre a interação com outras pessoas. Para A. Del Prette e Del Prette (2009) a expressividade de emoções pela face, como qualquer outro componente não-verbal, deve ser analisada a partir da complementaridade entre as propriedades topográficas e funcionais, onde a forma (topografia) é analisada em termos do impacto (função) sobre a qualidade e efetividade da interação social.

Os resultados indicam que todas as crianças apresentaram aquisições importantes em termos dos aspectos topográficos e funcionais da EFE e da EDE, que foram em geral mantidas ou aperfeiçoadas ao longo do tempo e para além do contexto de intervenção. Além disso, quanto aos dados da EDE, avaliada por meio de fotografias e filmagens, poucas diferenças foram observadas entre as duas formas de registro e aquelas que ocorreram foram, principalmente, na expressão de duas emoções que têm características topográficas semelhantes: medo e surpresa. Para estas duas emoções os dados da filmagem foram mais sensíveis, como destacam Ekman e Friesen (1975), para registrar as peculiaridades dos sinais faciais característicos de cada emoção e o contínuo da expressividade, em termos de inicio, meio e fim.

No caso do repertório de habilidades sociais, destaca-se que o programa de intervenção teve maior impacto exatamente sobre as classes que dependem de maior expressividade de emoções, tais como, empatia, assertividade, autocontrole, evitação de problemas, na versão SSRS-criança; autocontrole/civilidade e autocontrole passivo, na versão SSRS-pais; Autocontrole e cooperação com pares, na versão SSRS-professor (com ganhos para praticamente todas as crianças). Os dados sugerem, portanto, que o programa se mostrou mais efetivo no aprimoramento das classes de habilidades sociais que têm a expressão facial de emoções como um dos seus principais componentes.

É interessante destacar que não houve grande discrepância entre as crianças quanto aos ganhos obtidos com a intervenção, independente do comprometimento em termos deficiência visual. Assim, independente da deficiência visual, a EDE e EFE podem ser aprimorados quando são dispostas condições de intervenção apropriadas para isso. É importante ressaltar que essas condições de intervenção incluíram adaptações às especificidades de cada criança (forma de acesso às informações do ambiente e recursos requeridos em função do comprometimento visual), sugerindo a importância desse cuidado na efetividade da intervenção. Além disso, essa "homogeneidade" pode ser decorrente dos cuidados tomados na seleção dos participantes, uma vez que todas as crianças apresentavam um repertório de entrada bastante deficitário em habilidades sociais, EFE e EDE.

No caso das crianças deficientes visuais, entende-se que é imprescindível estabelecer, como condições diferenciadas, o acesso às informações do ambiente por vias alternativas, ou seja, outros canais sensoriais que não a visão, conforme recomenda Costa et al. (2005). Outros aspectos, igualmente significativos, podem ser destacados como possíveis ingredientes da intervenção efetuada no presente estudo. Um primeiro foi o de enfocar a EFE tanto em seus aspectos topográficos como funcionais: em termos topográficos, o programa buscou auxiliar as crianças a discriminarem e a apresentarem os sinais faciais característicos de cada emoção; em termos funcionais, buscou-se auxiliar as crianças a regularem a EDE de acordo com as classes de habilidades sociais e as demandas do contexto, estimulando-se constantemente suas análises da relação entre o comportamento socialmente competente, os antecedentes e os consequentes imediatos de modo a promover a discriminação e leitura do ambiente social em que os comportamentos ocorrem (A. Del Prette \& Del Prette, 2009). No caso das crianças com deficiência visual, os aspectos formais e funcionais da EFE têm sido enfatizados por vários pesquisadores (Anzano \& Rubio, 
Ferreira, B. C. \& Del Prette, Z. A. P. (2013). Programa de Expressividade Facial de Emoções e Habilidades Sociais de Crianças Deficientes Visuais e Videntes.

1995; Castanho, 1996; Costa, 2005; Tinti, 2003) que os identificam como deficitários e menos regulados e controlados pelas variáveis contextuais, culturais e pessoais.

Dado o delineamento adotado, que permitiu controlar, conforme Cozby (2003) e Kazdin (1982), alguns fatores aleatórios que ameaçam a validade interna (dados objetivos, avaliação contínua das variáveis dependentes, sondagens múltiplas de casos), os resultados alcançados neste estudo podem ser atribuídos aos ingredientes da intervenção. Vários pesquisadores do campo das habilidades sociais apresentam recomendações sobre esses cuidados, em geral referindo-se a delineamentos de grupo (Bolsoni-Silva, Del Prette, Montanher, Bandeira, \& Del Prette, 2006; Z. A. P. Del Prette \& Del Prette, 2011; Murta 2005; Rocha, Del Prette, \& Del Prette, 2008). Esses pesquisadores reconhecem a escassez de intervenções experimentais que tomam o sujeito como próprio controle e alguns apontam direções para uma avaliação individualizada, mesmo quando a intervenção ocorre em grupo, por exemplo, com o uso do método JT (Aguiar et al., 2009). Tais alternativas são particularmente pertinentes no caso de intervenções como a deste estudo, junto a crianças com necessidades educacionais especiais, como é o caso da deficiência visual.

Além da validade interna, o presente estudo produziu evidências de validade externa, em termos de manutenção e generalização do repertório de EDE. As estratégias de atribuição de tarefas de casa, ensino de descrição e análise funcional do comportamento, orientação a pais e professoras podem ter sido cruciais para essa generalização do repertório adquirido pelos participantes para outros ambientes e para sua manutenção no decorrer do tempo.

Não obstante os resultados positivos, o presente estudo possui algumas limitações. Uma delas se refere à amplitude do tema abordado neste artigo, que poderia suscitar uma análise com mais profundidade sobre os diferentes aspectos teóricos, metodológicos e práticos envolvidos nas pesquisas sobre expressões faciais de emoções e habilidades sociais.

Uma outra limitação foi o fato de não ter sido efetuada uma sondagem múltipla com igual número de avaliações pré-intervenção para os três grupos e com estabilidade da linha de base (Sidman, 1975), de modo a garantir maior validade interna às alterações produzidas pela intervenção. $O$ fato de não ter sido realizado um delineamento de linha de base múltipla entre participantes de cada subgrupo também pode ser considerada outra limitação desta pesquisa. Estudos futuros de replicação da intervenção aqui relatada, com esses e outros delineamentos mais sofisticados, seriam, portanto, bastante desejáveis.

Adicionalmente, pode-se apontar alguns encaminhamentos para futuras investigações como, por exemplo, a investigação mais detalhada dos ingredientes do programa. A identificação dos componentes críticos do programa é uma condição importante para o seu aperfeiçoamento e para a sua sistematização em protocolos que permitam sua replicação com integridade.
Um ingrediente que parece ter sido crucial e que poderia ser alvo de novas investigações foi o uso de estratégias para generalização dos resultados em termos de tarefas de casa e a entrega semanal de folhetos instrucionais aos pais e professoras, com informações sobre as habilidades alvo de cada módulo da intervenção, importância deste repertório para a criança e dicas para os significantes estimularem o desenvolvimento social e emocional da criança; entre outros.

O envolvimento de pais e professoras é particularmente importante para que estes fiquem mais sob controle dos comportamentos do seu filho/aluno, consequenciado-os, refinando-os, propiciando ambientes mais sensíveis a seus desempenhos e aumentando a probabilidade de manutenção no decorrer do tempo e generalização para outros contextos, além do familiar e escolar.

\section{Referências}

Aguiar, A. A. R. de, Aguiar, R. G., \& Del Prette, Z. A. P. (2009). Calculando a significância clínica e o índice de mudança confiável em pesquisa-intervenção. São Carlos, SP: Editora da Universidade Federal de São Carlos.

Anzano, S. M., \& Rubio, J. M. L. (1995). Entrenamiento en habilidades sociales a niños con ceguera congénita. In F. G. Rodrígues, J. M. L. Rubio, \& L. J. Expósito (Eds.), Habilidades sociales y salud (pp. 147-183). Madrid, España: Pirámide.

Bandeira, M., Del Prette, Z. A. P., Del Prette, A., \& Magalhães, T. (2009). Validação das Escalas de Habilidades Sociais, Comportamentos Problemáticos e Competência Acadêmica (SSRS-BR) no ensino fundamental. Psicologia: Teoria e Pesquisa, 25, 271-282.

Bee, H. (1996). A criança em desenvolvimento. Porto Alegre, RS: Artes Médicas.

Bolsoni-Silva, A. T., Del Prette, Z. A. P., Montanher, A. R. P., Bandeira, M., \& Del Prette, A. (2006). A área das habilidades sociais no Brasil: Uma análise dos estudos publicados em periódicos. In M. Bandeira, Z. A. P. Del Prette, \& A. Del Prette (Eds.), Estudos sobre habilidades sociais e relacionamento interpessoal (pp. 17-45). São Paulo, SP: Casa do Psicólogo.

Castanho, A. R. S. P. (1996). A face de crianças deficientes visuais: Expressões de emoções e percepção social de seus sorrisos (Tese de doutorado, Programa de Pós-Graduação em Psicologia Experimental, Universidade de São Paulo, SP, Brasil).

Castanho, A. R. S. P., Moifrel, A. C. B., Severiano, E., \& Ribeiro, V. R. (2003). Gestos não-verbais espontâneos e canais de expressão emocional voluntária em deficientes visuais. Revista Benjamin Constant, 26.

Celeste, M. (2006). Play behaviors and social interactions of a child who is blind: In theory and practice. Journal of Visual Impairment \& Blindness, 100, 75-90.

Costa, C. S. L. (2005). Habilidades sociais de criança cega e vidente, crenças e práticas educativas da mãe: Um estudo de caso com gêmeas idênticas (Dissertação de mestrado, Programa de Pós-Graduação em Educação Especial, Universidade Federal de São Carlos, SP, Brasil).

Costa, C. S. L., Del Prette, A., Cia, F., \& Del Prette, G. (2005). Sentimentos expressos por gêmeas idênticas (cega e vidente) Comparando três procedimentos de identificação. Psico, 36, 205-211. 
Cozby, P. C. (2003). Métodos de pesquisa em ciências do comportamento. São Paulo, SP: Atlas.

D' Allura, T. (2002). Enhancing the social interaction skills of preschoolers with visual impairments. Journal of Visual Impairment \& Blindness, 9, 577-584.

Del Prette, A., \& Del Prette, Z. A. P. (2001). Psicologia das relações interpessoais: Vivências para o trabalho em grupo. Petrópolis, RJ: Vozes.

Del Prette, A., \& Del Prette, Z. A. P. (2009). Componentes não-verbais e paralinguísticos das habilidades sociais. In Z. A. P. Del Prette \& A. Del Prette (Eds.), Psicologia das habilidades sociais: Diversidade teórica e suas implicações (pp. 149-188). Petrópolis, RJ: Vozes.

Del Prette, Z. A. P., \& Del Prette, A. (2005). Habilidades sociais na infância: Teoria e prática. Petrópolis, RJ: Vozes.

Del Prette, Z. A. P., \& Del Prette, A. (2006). Avaliação multimodal de habilidades sociais em crianças: Procedimentos, instrumentos e indicadores. In M. Bandeira, Z. A. P. Del Prette, \& A. Del Prette (Eds.), Estudos sobre habilidades sociais e relacionamento interpessoal (pp. 47-68). São Paulo, SP: Casa do Psicólogo.

Del Prette, Z. A. P., \& Del Prette, A. (2008) A significância clínica e mudança confiável: A efetividade das intervenções em Psicologia. Psicologia: Teoria e Pesquisa, 24, 497-506.

Del Prette, Z. A. P., \& Del Prette, A. (2011). Práticas baseadas em evidência e treinamento de habilidades sociais. In A. Del Prette \& Z. A. P. Prette (Eds.), Habilidades sociais: Intervenções efetivas em grupo (pp. 57-82). São Paulo, SP: Casa do Psicólogo.

Ekman, P. (1982). Emotion in the human faces. Cambridge, MA: Cambridge University Press.

Ekman, P. (2004). Emotions revealed: Recognizing faces and feelings to improve communication and emotional life. New York: Henry Holt and Company.

Ekman, P., \& Friesen, W.V. (1975). Unmasking the face: A guide to recognizing emotions from facial expressions. Cambridge, MA: Malor Books.

Ferreira, B. C. (2008). Identificação de sentimentos e desempenho empático em crianças deficientes visuais e videntes: Um estudo comparativo e multimodal (Dissertação de mestrado, Programa de Pós-Graduação em Educação Especial, Universidade Federal de São Carlos, SP, Brasil).

Ferreira, B. C. (2012). Expressões faciais de emoções de crianças deficientes visuais e videntes: Avaliação e intervenção sob a perspectiva das habilidades sociais (Tese de doutorado, Programa de Pós-Graduação em Psicologia, Universidade Federal de São Carlos, SP, Brasil).

Galati, D., Scherer, K. R., \& Ricci-Bitti, P. E. (1996). Voluntary facial expression of emotion: Comparing congenitally blind with normally sighted encoders. Journal of Personality and Social Psychology, 73, 1363-1380.

Godoy, A. M. A. (2007). Indicadores de empatia em crianças com baixa visão e em seus pais: Um estudo de caso (Dissertação de mestrado, Programa de Pós-Graduação em Educação Especial, Universidade Federal de São Carlos, SP, Brasil).

Hueara, L., de Souza, C. M. L., Batista, C. G., Melgaço, M. B., \& Tavares, F. S. (2006). Os faz-de-conta em crianças com deficiência visual: Identificando habilidades. Revista Brasileira de Educação Especial, 12, 351-368.

Kazdin, A. E. (1982). Single-case research designs: Methods for clinical and applied settings. New York: Oxford University Press.
Laplane, A. L. F., \& Batista, C. G. (2008). Ver, não ver e aprender: A participação de crianças com baixa visão e cegueira na escola. Cadernos Cedes, 28, 209-227.

Matsumoto, D., \& Willingham, B. (2009). Spontaneous facial expressions of emotion of congenitally and noncongenitally blind individuals. Journal of Personality and Social Psycho$\log y, 96(1), 1-10$.

Murta, S. G. (2005). Aplicações do treinamento de habilidades sociais: Análise da produção nacional. Psicologia: Reflexão e Crítica, 18, 283-291.

Ortega, M. P. P. (2003). Linguagem e deficiência visual. In M. B. Martín \& S. T. Bueno (Eds.), Deficiência visual: Aspectos psicoevolutivos e educativos (pp. 77-95). São Paulo, SP: Santos.

Otta, E. (1994). O sorriso e seus significados. Petrópolis, RJ: Vozes.

Otta, E., Ribeiro, F. L., \& Bussab, V. S. R. (2003). Inato versus adquirido: A persistência da dicotomia. Revista de Ciências Humanas, 34, 283-311.

Philippot, P., Feldman, R. S., \& McGee, G. (1992). Nonverbal behavioral skills in a educational context: Typical and atypical populations. In R. S. Feldman (Ed.), Apllications of nonverbal behavioral theories and research (pp. 191-213). Hillsdale, NJ: Lawrence Erlbaum.

Riggio, R. E. (1992). Social interaction skills and nonverbal behavior. In R. S. Feldman (Ed.), Apllications of nonverbal behavioral theories and research (pp. 3-30). Hillsdale, NJ: Lawrence Erlbaum.

Rocha, M. M., Del Prette, Z. A. P., \& Del Prette, A. (2008). Placebo na pesquisa psicológica: Algumas questões conceituais, metodológicas e éticas. Revista Brasileira de Terapias Cognitivas, 4, 39-55.

Schachner, D. A., Shaver, P. R., \& Mikulincer, M. (2005). Patterns of nonverbal behavior and sensitivity in the context of attachment relationships. Journal of Nonverbal Behavior, 29, 141-169.

Sidman, M. (1975). Táticas de pesquisa científica. São Paulo, SP: Brasiliense.

Tinti, C. (2003). Spontaneous facial expressions in congenitally blind and sighted children aged 8-11. Journal of Visual Impairment \& Blindness, 97, 418-428.

Tröster, H., \& Brambring, M. (1992). Early social-emotional development in blind infants. Child: Care, Health and Development, 18, 207-227. 